\title{
Food Poisoning with Special Reference to the Foods Concerned
}

\section{By J. Cruickshank, Department of Bacteriology, University of Aberdeen}

'The term 'food poisoning' as generally used refers to outbreaks of acute illness (sickness, vomiting, diarrhoea) occurring shortly after a meal and affecting as a rule a number of individuals such as a family, a small community, or a group of persons who have been gathered together and have partaken of the same meal. The symptoms occur within a few hours, may be mild or severe and are occasionally followed by a fatal issue. To the bacteriologist these food poisonings are in most cases infections of the alimentary tract associated with acute inflammation of the lining of the stomach and upper intestine, a condition of acute gastro-enteritis. Bacteriological examination of the food or vomited material usually reveals the presence of bacteria of the salmonella group, or certain strains of staphylococci which are distinguished by their power to produce 'enterotoxin'. In a few outbreaks dysentery organisms occur and the symptoms are referable to the large intestine, with severe diarrhoea. In exceptional cases, rarely in this country, the cause of the illness is the presence in the food of powerful toxins resulting from the growth of the anaerobic organism Clostridium botulinum. Food poisoning may occur from the presence of metallic poisons, e.g. zinc, bismuth, lead, copper, or arsenic derived from the food containers during storage, cooking or distribution, or by the ingestion of poisonous plants, especially the berries of the deadly nightshade, foxglove, or henbane, or the poisonous toadstools. By far the most common causes are bacterial infections caused by the salmonella group and by the presence in food of the enterotoxin-producing strains of staphylococci. It should be noted that many other human diseases may be caused by the ingestion of infected foodstuffs, especially milk and its products. These diseases are not listed as food poisonings owing to the time which separates the taking of the food and the development of the first signs and symptoms. Into this category fall the enteric fevers typhoid and paratyphoid, Malta and abortus fevers, tuberculosis, streptococcal sore throat, and diphtheria. 'The contamination of the food occurs, in many cases, from human sources during the preparation or distribution; in others the pathogenic organism is present in an infected animal and the flesh or milk contains many organisms. With the salmonella infections either of these sources may be found, whereas in staphylococcal infections the organism is almost invariably derived from contact of the food material with the human subject. Staphylococci are common in the nose and throat of many persons, and the hands of workers are readily contaminated, especially from nasal infections. Localized staphylococcal lesions of the fingers are not infrequent and may be the ready means of adding organisms to food material. It is a fortunate circumstance that only relatively few strains of staphylococci have the property of producing enterotoxin, otherwise food poisoning by these bacteria would be much more frequent.

'The term 'food poisoning' was originally applied to these illnesses in the belief that substances called 'ptomaines' produced in the course of putrefaction of food were responsible. The explosive nature of many of the outbreaks naturally suggests a true poisoning, but, with the exception of botulism, they are true infections or the result of 
ingestion of preformed bacterial toxins. Owing to the short interval, 2-3 hr., in some outbreaks, between participation in a meal and the onset of nausea and vomiting, it is suggested that even in salmonella infections preformed bacterial toxin is also present in the food and relatively resistant to the temperatures of cooking. No substantial proof of the existence of such substances has been produced, in spite of much laboratory investigation.

Salmonella organisms are widely distributed in nature as infective agents of rodents, fowls, ducks, and domestic animals, and within recent years more than 200 strains have been distinguished by antigenic analysis. In human cases of food poisoning from salmonella infection the source has not commonly been the human chronic carrier as it frequently is in enteric infections. More often contamination of the food occurs by contact with infected meat or eggs, or by the faeces of infected rats or mice, in the latter case usually during the storage of cooked or partly cooked food allowed to cool slowly. During the war, when vast quantities of dried eggs were imported from America, many salmonella strains new to this country were introduced. It is reported that $10 \%$ of the samples of spray-dried eggs contained these organisms. The need for thorough cooking was well emphasized, but outbreaks of food poisoning were not infrequent. The organism may be found even in the interior of duck eggs and the only reliable way of ensuring sterility is boiling for $8 \mathrm{~min}$. The eggs of fowls also may be infected during their formation in the oviduct, but more frequently the shell is contaminated with the faeces, and under certain conditions organisms may penetrate the shell. It is important not to disturb the albumin layer on the outside of the shell, and to avoid exposure of the egg to moist faecal material as the egg cools. Storage at low temperature $\left(5^{\circ}\right)$ is important in order to prevent growth of the infecting organism.

In this country salmonella infections of fowls are also very common in the form of bacillary white diarrhoea, caused by Salmonella pullorum, and ducks suffer from keel disease, caused by Salm. anatum. Neither of these organisms, however, contributes materially to human food poisoning.

Pigs, cattle, horses, sheep, rats and mice suffer from natural salmonella infections, pigs especially. Infected rodents may carry the infection for long periods in the internal organs and act as chronic carriers. Certain strains are common in pigs and cattle, and the recognition of the particular strain may indicate the probable source, but in most cases establishment of the antigenic type gives little indication of the source. Beef and pork may be heavily infected in the natural salmonella diseases of cattle and pigs, and infections of cow's milk may give rise to extensive outbreaks of acute gastro-enteritis.

In recent years staphylococcal food poisoning has frequently been reported and bids fair to replace salmonella infection as the most common cause of food poisoning. Incriminated food is heavily infected with staphylococci, and there is evidence that a toxin relatively stable to heat is produced. From time to time staphylococci may be present in large numbers in the nose and throat and on the skin. In the laboratory some strains are capable of producing filtrates which induce nausea, sickness and vomiting in human volunteers. Intraperitoneally some filtrates may induce symptoms in young kittens or young monkeys. The property. of producing enterotoxin is present in 
relatively few strains under laboratory conditions, and it is reasonable to suppose that the conditions in certain foodstuffs are more suitable for development of the enterotoxin. 'The nature of this substance is not yet clear. Studies in Aberdeen of the microbial flora of fish thoroughly cooked (autoclaved), minced and spread on shallow trays preparatory to drying showed that the sterile hot material became heavily infected during the process of mincing and spreading, and that Staphylococcus aureus was present in large numbers under the conditions of manufacture, in which large quantities of fish were used. Even after drying, staphylococci were found by culture in considerable numbers. These organisms were clearly derived from the workers. In the absence of suitable, reliable tests for enterotoxin the risk of poisoning had to be taken during the war period. There was no evidence that the conditions were suitable for enterotoxin production in the relatively short time between autoclaving the product and the final drying, in spite of the favourable conditions for proliferation of the staphylococci.

The conditions for the development of enterotoxin are most favourable when meat, fish, pork or other flesh is much handled, as in mincing for production of brawn, meat jellies and pies. The large exposure of surfaces, the previous cooking at temperatures short of achieving sterility, the gradual cooling are all such as to favour gross bacterial contamination. 'The preparation of food on a large scale, as for a large company, is apt to throw a strain on hotel and restaurant kitchens, and in practice often leads to precooking of foods with subsequent reheating before it is presented at table. Such conditions are ideal for infection with salmonella from the faeces of rats and mice, or for staphylococcal growth.

'Thus all meat foods of the minced type, sausages, pressed beef, brawn, meat pies and pasties are suspect. Milk, cream and cream cakes are regularly found to be incriminated and, because of their wide distribution in a community, may be the cause of large epidemics of food poisoning. A review of epidemics reported during the last ro years shows a great increase over previous years in the outbreaks of staphylococcal poisoning. Trifle, ice-cream, cow's milk, goat's milk, cheese, meat pies, brawn, meat-pie jelly, bacon and potato pie, custard filling, cream puffs, cream-custard cakes, smoked ham, cooked ham, tinned ham, cooked chicken, cooked salmon and pickled-meat sandwiches have been reported as the vehicles of staphylococcal poisoning. In almost all cases nasal carriers of Staph. aureus were the agents of infection, but septic hands of cooks and milkers, as well as milk from cows with mastitis, were likely sources. The salmonella infections in the same period were associated with eggs, pigs fed on garbage containing poultry entrails and carcasses, butter, cream cakes, cheese (a dead mouse in the cheese vat), roast pork, jellied veal, pressed beef, imported chilled turkey, infected turkey meal, smoked fish and canned camel (in Egypt). Salm. typhi-murium was reported as frequently as staphylococcus. Strains of Salm. enteritidis were reported from canned beef hash, calf meat, cheese, sausage, milk, cream custard, rice cakes, and various meats and meat pastes. In one of these outbreaks, due to infected cheese, no less than eighty-two people became chronic carriers of Salm.jena. In another outbreak the same cloth was used in the slaughter-house to wipe down an infected pig and healthy carcasses. In only very few outbreaks was a human carrier implicated. Between the years 1942 and 1944 no less than twenty-four types of salmonella new to this country were 
recovered from spray-dried eggs of American origin. Some of these types were found also in the mesenteric glands of pigs fed on spray-dried eggs condemned as unfit for human consumption. Other strains have been reported in recent years.

In addition to the salmonella and staphylococcal poisonings, severe outbreaks of bacillary dysentery have occurred, especially through the medium of milk. In such cases the infection can usually be traced to the human subject.

Many organisms other than salmonella and staphylococcus are recorded as probable causes of food poisoning. Some outbreaks have been related to the presence in food of Streptococcus viridans, Proteus, Bacterium coli, Bacillus subtilis and related organisms. Laboratory proof of the production of toxic substances by these organisms is lacking, and acceptance of these organisms as causative agents would almost lead one back to the original theory of 'ptomaine' poisoning.

An enormous amount of food is produced, especially in America, by the canning process and is consumed by people all over the world. It is a remarkable testimony to these products that food poisoning is so seldom reported. Sterility of the material is usually possible, or at any rate it can be heated sufficiently to destroy non-sporing bacteria. 'The only organism of importance, $C l$. botulinum, whose spores may be exceptionally heat-resistant, has seldom been the cause of food poisoning in this country. Since the Loch Maree outbreak it has been encountered on only three occasions and has affected few people. The foods responsible were potted duck paste, vegetarian nutbrawn and home-made pies. Botulism has been reported much more frequently in America and on the Continent of Europe. It is associated most frequently with homecanned vegetables, not with the acid fruits. The toxin develops in the food before it is eaten; the organism itself does not grow in the affected subjects. The organism is present in soil, especially virgin soil, and its spores are sometimes extremely resistant to heating. Special conditions are necessary for the development of the spores: anaerobic environment; spores in sufficient numbers, possibly; insufficient cooking; and a suitable hydrogen-ion concentration. The foods which have produced cases of botulism in America are string beans, olives, spinach, maize, peas and salmon. In Europe sausages, ham, preserved meats, potted goose or duck, brawn and salted fish have been the vehicles of the disease. In many cases the appearance of the contents of the cans has been indicative of spoilage.

\section{Vitamin $\mathrm{C}$ and Immunity}

\section{By G. H. Bourne, London Hospital Medical College, Whitechapel, London, E. I}

The term immunity means complete insusceptibility to infection, but we are not perhaps as much interested in this absolute condition as in relative immunity: in other words, a variable which may be anywhere between zero and infinity. The word immunity is better replaced by the word resistance, so that what I should talk about in this paper is whether vitamin $\mathrm{C}$ plays any part in improving the relative resistance of the body to infective agents. 